\title{
Germanica
}

GERMANICA

39 | 2006

La nouvelle génération d'écrivains de langue allemande

\section{Les nouvelles de Michael Kleeberg : littérature et réalité}

Michael Kleebergs Novellen: Literatur und Realität

\section{Alain Cozic}

\section{OpenEdition}

\section{Journals}

Édition électronique

URL : http://journals.openedition.org/germanica/307

DOI : 10.4000/germanica.307

ISSN : 2107-0784

\section{Éditeur}

Université de Lille

\section{Édition imprimée}

Date de publication : 1 décembre 2006

Pagination : $27-42$

ISBN : 2-913857-18-3

ISSN : 0984-2632

\section{Référence électronique}

Alain Cozic, «Les nouvelles de Michael Kleeberg : littérature et réalité », Germanica [En ligne], 39 | 2006, mis en ligne le 19 février 2010, consulté le 06 octobre 2020. URL : http://journals.openedition.org/ germanica/307 ; DOI : https://doi.org/10.4000/germanica.307

Ce document a été généré automatiquement le 6 octobre 2020.

(c) Tous droits réservés 


\title{
Les nouvelles de Michael Kleeberg : littérature et réalité
}

\author{
Michael Kleebergs Novellen : Literatur und Realität
}

\author{
Alain Cozic
}

1 Michael Kleeberg est né en 1959 à Stuttgart, a grandi à Böblingen, petite ville sise entre Tübingen et Stuttgart, et Hambourg. Il a séjourné à Rome, Berlin et Amsterdam, puis de 1986 à 1994 à Paris où il a travaillé pour une agence de publicité. Après avoir prolongé son séjour en France jusqu'en 2000, il vit depuis à Berlin. Il a traduit en allemand des auteurs français comme Yves Simon, Xavier Hanotte, Pascal Bruckner, Paule Constant, Virginie Despentes, mais aussi Barbey d'Aurevilly et Proust ${ }^{1}$.

2 Il a publié jusqu'à présent quatre romans : Der Saubere Tod. Roman, Proteus der Pilger. Roman, Ein Garten im Norden. Roman, Der König von Korsika. Roman. Ein Garten im Norden, notamment, lui a valu un succès retentissant. Sa dernière œuvre en date est un journal de voyage, Das Tier, das weint. Libanesisches Reisetagebuch, rédigé à l'occasion d'un séjour de quatre semaines à Beyrouth en $2003^{2}$.

3 C'est au nouvelliste que la présente contribution va être consacrée. Trois publications illustrent ce volet de la production de Kleeberg: un recueil, Böblinger Brezeln. Stories, avec lequel il a commencé sa carrière littéraire en 1984, une longue nouvelle de près de 150 pages, publiée en 1995, Barfuß. Novelle, et un autre recueil en date de 1997, Der Kommunist vom Montmartre und andere Geschichten ${ }^{3}$. Les lignes qui suivent proposent un parcours de lecture à travers les « histoires » composant ce dernier ensemble. Celui-ci nous paraît en effet présenter un nouvelliste au sommet de son art, tant sur le plan des thématiques abordées que sur celui des techniques d'écriture. La nouvelle comme genre, avec les spécificités qui sont les siennes en tant que genre et qui seront analysées elles aussi, atteint ici une forme accomplie. Mais cette étude des nouvelles de Michael Kleeberg aura également atteint son objectif si elle est parvenue à convaincre le lecteur d'aborder - aussi - les romans de cet auteur à n'en pas douter important !

Böblinger Brezeln, déjà, laissait bien augurer des qualités, de l'originalité et de la force évocatrice du nouvelliste Kleeberg. Les quatorze (short) «stories» constituant le 
recueil sont en effet autant de tranches de vie, d'instantanés saisis dans l'existence souvent sordide de personnages à la dérive, marginaux ayant perdu toute attache et tout repère, solitaires en mal de rencontres. En quelques traits révélateurs, Kleeberg sait faire surgir un milieu et dérouler le destin, dérisoire ou pathétique, de ceux qui le peuplent ; l'art du nouvelliste est déjà là en germe.

D'une autre ampleur est Barfuß, l'histoire de Arthur K. - le début, outre le nom abrégé du personnage, fait songer à Kafka -, publiciste parisien qui, par hasard et par erreur, en consultant le Minitel est orienté vers un site sado-masochiste. Poussé par sa curiosité, il se laisse entraîner, après avoir répondu à une demande, à des jeux pervers de plus en plus violents, sacrifiant sa vie professionnelle et sa vie personnelle, jusqu'à l'ultime épisode où il se fait crucifier - au sens littéral du terme - par son persécuteur. C'est, en dépit d'une existence où tout paraît lui sourire, sans doute un vide essentiel qui pousse le personnage à de telles extrémités, cherchant dans la soumission, puis dans l'anéantissement final, une forme de reconnaissance à ses propres yeux, sinon de liberté, d'extase dans la douleur, d'extase extrême dans la mort demandée et acceptée. Malgré sa sordidité et le malaise que l'on éprouve à sa lecture, on peut aussi voir dans cette nouvelle une réflexion sur le sens (le non sens?) de la vie.

Les nouvelles de Der Kommunist vom Montmartre vont prolonger, selon d'autres modalités, cette réflexion, tout en interrogeant encore, pour certaines d'entre elles, les tréfonds de l'individu, la part sombre tapie en tout un chacun.

\section{Littérature et réalité de la vie}

7 Literatur: ainsi est intitulée l'une de ces "histoires ». Le titre est aussi simple et lapidaire que le sujet est complexe, puisque la " littérature », en effet, est au cœur de ce récit, et sous les multiples facettes qu'elle peut offrir : le rôle qu'elle joue pour celui qui la pratique et en fait sa raison d'être, les rapports que les lecteurs entretiennent avec elle, les interprétations qu'elle suscite chez ceux dont c'est le métier de l'analyser critiques ou universitaires -, les comportements qu'elle génère chez les éditeurs qui la publient, d'autres manifestations encore, comme les prix littéraires décernés en son nom, sinon en son honneur.

8 L'organisation d'un tel concours littéraire lance le récit; la manifestation a lieu dans une petite ville au sud-est de l'Autriche près de la frontière qui fut autrefois yougoslave et qui est désormais slovène (le prix Ingeborg Bachmann à Klagenfurt pour lequel Kleeberg lui-même a concouru en 1993 ?). Aucun des différents participants ne sort indemne du portrait qui est brossé de lui : les membres du jury, assimilés à des juges décernant titres et médailles lors de jeux olympiques ou à des procureurs de tribunal révolutionnaire, qui raisonnent en catégories nettement tranchées entre "récits réalistes » et "textes expérimentaux », leur préférence allant systématiquement aux seconds ... d'autant plus facilement qu'ils ne les comprennent pas, les auteurs, en quête de reconnaissance ou de contrat éditorial, recherchant les interviews des journalistes conviés, appréciant l'invitation tous frais payés et le luxe du quatre étoiles qui les héberge, les spectateurs eux-mêmes, dont la catégorie la moins représentée est constituée par les lecteurs véritablement passionnés de littérature, curieux de découvrir des œuvres et des auteurs qu'ils ne connaissent pas, les autres se distribuant en voyeurs alléchés par le spectacle de juges étrillant des écrivains n'en pouvant mais, 
et en provinciaux heureux que télévision et autres médias se soient donné rendez-vous en leur bonne ville pour en rompre l'espace de quelques jours la lancinante monotonie.

Kleeberg propose en ces pages, avec une verve à l'évidence jubilatoire, une certaine image du monde littéraire, de ses fastes - relatifs - et de ses frasques - bien réelles -, où celui qui est supposé être l'acteur majeur - l'auteur, qui, il est vrai, en quête de reconnaissance, sacrifie au rite - est surtout ravalé au rang de marionnette, livré en pâture au cours d'un rituel qui relève de la foire aux vanités, des jeux du cirque, d'un jeu de massacre.

Mais la nouvelle - et c'est là son intérêt essentiel - présente simultanément et par effet de contraste une autre image, moins du monde littéraire que de la littérature et de ceux qui, sincères et convaincus, lui donnent une vie réelle. Simultanément se tient en effet, dans un village de montagne frontalier, à 35 kilomètres de la ville du concours, une autre rencontre littéraire organisée par une petite maison d'édition qui se consacre aux écrivains originaires des pays ayant constitué l'ancien empire austro-hongrois. Cette manifestation ne suscite guère l'intérêt des participants au concours; seuls trois des douze auteurs invités, deux des six membres du jury et un seul des cinquante journalistes décident de s'y rendre, la motivation de chacun n'ayant du reste qu'un rapport lointain avec la nature de la manifestation. L'une de ces personnes, toutefois, détonne et c'est autour d'elle, de la situation dans laquelle elle se trouve et de ses réactions, que Kleeberg construit sa nouvelle, c'est cet écrivain, Gerhard Schrader, qui lui aussi a participé au concours, s'y est fait taxer d'écrivain « réaliste» et en conséquence dûment malmener, qui confère au récit toute sa signification.

11 Employé au service juridique d'une banque privée, Schrader s'est aussi essayé à la littérature, non sans succès. Ce ne sont pas les critiques dont il vient de faire l'objet de la part du jury qui préoccupent Schrader au moment où il décide de se rendre à la rencontre littéraire, c'est l'échec de sa vie personnelle, dont deux semaines auparavant il a pris conscience lorsque sa femme l'a quitté pour un autre, emmenant avec elle leur fille. Dix années de vie commune et un avenir qui paraissait assuré et heureux se sont ainsi brusquement effondrés. Schrader fait à cette occasion l'expérience du vide et de la souffrance, ce qui l'amène à s'interroger aussi sur le sens de la littérature face à semblables événements que produit la vie. Il ne lui sera d'aucune utilité, à lui l'écrivain, de tenter d'écrire pour dire, contenir, surmonter sa douleur, il n'en a du reste aucunement envie, et il n'écrira plus. Aucune fiction ne serait à même d'exprimer la souffrance éprouvée, et surtout elle ne servirait à rien. Les mots inventés ne diront jamais les maux ressentis. Echec de la littérature donc pour transcrire cette réalité-là, qui est essentielle puisqu'elle est de souffrance. Inadéquation du verbe puisque ce que l'on éprouve relève précisément de l'indicible.

12 Kleeberg semble ici, par personnage de fiction interposé - mais celui-ci n'est-il pas proche de l'Auteur?-, en lui prêtant de telles réflexions, récuser en quelques phrases simples mais pesées et ciselées toute une conception de la littérature qui veut voir, dans l'œuvre écrite, pour celui qui la rédige et met en mots les souffrances ressenties, une manière de thérapie, conception qui confère à l'acte d'écrire une valeur cathartique. Toute une littérature du moi, et en l'occurrence du moi souffrant, serait de la sorte implicitement battue en brèche, et implicitement rejetés ces textes dans lesquels les auteurs se plaisent et se complaisent à confier à la feuille blanche les tourments qui les déchirent, espérant ou feignant de croire que le fait de transcrire leurs maux sur le papier pourra les atténuer un peu. Décidément, semble dire Kleeberg, 
la littérature pèse d'un poids tout relatif face à la réalité de la vie, dès lors que celle-ci est dévastatrice pour celui qui doit en supporter les désagréments. La question débattue ici n'est pas anodine, qui assigne à la littérature, à l'acte d'écrire, à l'œuvre littéraire leurs limites, circonscrit leur portée, sinon leur raison d'être. Mais il est vrai aussi que, pour ce faire, l'écrivain Kleeberg propose lui-même une œuvre de fiction une œuvre littéraire - éminemment construite et écrite! Autoréflexivité singulière et distanciée d'un texte qui fixerait de la sorte les bornes à une littérature dont il est pourtant un exemple des plus convaincants!

Si ces réflexions de Schrader, mesurées à l'aune de son expérience personnelle, précisent les relations entre la littérature et la réalité, paraissant disqualifier la première dès lors que la seconde impose avec force ses lois, la deuxième partie de la nouvelle, qui relate une autre expérience de Schrader, semble à l'inverse redonner à la littérature tout son crédit, montrer à quel point elle est nécessaire, démontrer comment elle peut et doit transcrire le réel.

14 À la rencontre-lecture organisée dans le village frontalier ont été invités trois auteurs, mais deux seulement sont présents, un romancier croate et un poète bosniaque. Le troisième, qui vit à Sarajevo et avait promis de venir, a disparu, personne n'a plus de nouvelles de lui depuis trois jours. L'année et l'époque, l'origine respective des deux écrivains présents et de celui qui n'est pas là, la disparition de ce dernier sont autant d'éléments qui précisent le contexte : nous sommes au cœur de la crise yougoslave qui enflamme les Balkans. Au moment où les deux écrivains se rencontrent, au printemps 1993, le cessez-le-feu autour de l'enclave musulmane de Srebrenica a été instauré. L'actualité a montré en son temps l'atrocité de cette guerre, dont la composante ethnique est essentielle. Les deux auteurs invités se font l'écho de ce conflit, chacun à sa manière et selon sa personnalité, par les extraits de leurs œuvres qu'ils lisent devant un public clairsemé, largement indifférent en outre, si l'on excepte l'éditeur et Schrader.

Le romancier croate lit des extraits de son œuvre dans sa langue maternelle, ne maitrisant pas l'allemand ; à la fin de sa lecture, l'éditeur traduit. Mais cette traduction est superflue. Par sa manière de conter, ses talents d'orateur, ses mimiques et ses gestes, ses intonations de voix, la magie de ses mots, l'écrivain a su faire comprendre à ses auditeurs, à Schrader en tout cas, le contenu de son texte, l'histoire de son pays dévasté. C'est, par le truchement des mots qui constituent son matériau, tout le pouvoir de la littérature qui s'exprime ici, la force évocatrice de la langue qui n'a plus besoin d'être traduite pour être comprise, qui dit la réalité, se fait le reflet exact d'un monde dévasté par les tempêtes de l'Histoire. Un autre rapport entre littérature et réel: différent de celui qu'évoquait Schrader lorsqu'il songeait à sa situation personnelle. À l'impuissance constatée et déplorée de la littérature pour rendre compte des douleurs ressenties succède désormais et s'oppose le pouvoir des mots qui, quel que soit l'idiome, quand bien même celui-ci ne serait pas maîtrisé par ceux qui l'écoutent, transcrivent et font comprendre d'autres souffrances.

Le poète bosniaque, quant à lui, est un rescapé des combats qui ont fait rage à Sarajevo. Les poèmes qu'il récite, ses "Kellergedichte", disent eux aussi, du point de vue bosniaque, les malheurs de la guerre, les affres de la vie quotidienne dans une ville livrée aux combats. Pouvoir de la littérature de nouveau, force de la langue poétique cette fois, pour exprimer et faire connaître la réalité, cette réalité-là ${ }^{4}$. 
17 L'éditeur lira encore, après l'intervention du poète, les textes de l'écrivain absent, courtes proses qui, elles aussi, décrivent avec concision la vie dans une ville assiégée.

Ces trois lectures, qu'il a écoutées avec attention, ont sur Schrader un effet inattendu. Lui qui ne croyait plus au pouvoir de la littérature, qui voyait entre elle et la vie une dichotomie irréductible, décide de ne pas repartir du village avec ceux qui l'ont accompagné, mais de se rendre seul et à pied de l'autre côté de la montagne où la guerre sévit toujours, pour «aller voir de l'autre côté, après quatre ou cinq jours de marche, ce qui, au milieu de l'embrasement de la guerre civile, pouvait bien faire naître le langage en lieu et place du silence » $(K, \mathrm{p} .217)$. Comme un voyage initiatique en définitive qu'il accomplirait de la sorte, lui l'écrivain qui ne veut plus écrire, fort de l'expérience édifiante qu'il vient de faire en écoutant ses trois confrères, afin de se rendre compte de visu et in situ comment littérature et réalité peuvent coller l'une à l'autre, la première se devant d'être, avec les modes d'expression des plus variés dont elle dispose, le reflet précis, conforme, parfait de l'autre, afin, en somme, d'abolir la dichotomie dans un premier temps pointée. Il n'ira pas jusqu'au bout de ce voyage, aura mal évalué les distances et préjugé de ses forces, sera retrouvé in extremis dans la montagne par un hélicoptère envoyé à sa recherche. La presse locale, relatant l'événement, tournera l'initiative de Schrader en dérision, la considérant comme l'une des lubies délirantes qui, traditionnellement, traversent l'esprit des participants au concours littéraire en mal de notoriété.

19 Malgré cette tentative avortée, en dépit de la chute de la nouvelle qui donne le beau rôle, rôle facile, aux représentants des médias, Kleeberg pose dans ce récit, très représentatif de la manière dont cet auteur appréhende et exprime le monde qui est le sien, le problème des rapports entre littérature et réalité. C'est l'indispensable présence de la littérature qui est ici mise en avant, pour éviter que ne s'installe le silence. La question soulevée est donc éternelle, celle du rôle de la littérature, de son aptitude à dire, à décrypter, sinon à dénoncer le réel, la question de la fonction de l'écrivain, de l'intellectuel. «Wozu Dichter in düsterer Zeit ? » pour le dire encore comme Hölderlin.

Literatur propose une réponse à cette question, cette nouvelle a, dans le recueil, valeur paradigmatique. Les autres récits sont des variations par rapport à la réponse ici apportée.

\section{Réalités allemandes}

Chez cet écrivain né en 1959, appartenant à une génération qui n'a connu ni le national-socialisme ni la guerre, le passé allemand, cette réalité allemande-là, pourtant ne sont pas absents. C'est à ce passé, brusquement, que doit faire face le narrateur de Der Vater von Lise, lorsque Lise, qui est juive, lui conte l'histoire de ses grands-parents déportés et gazés à Auschwitz. Ils habitaient Paris, malgré toutes les mises en garde et objurgations, celles de leur fils, le père de Lise, notamment, ils ont refusé de fuir et finirent par être arrêtés. C'est «le père de Lise ", ainsi que le suggère le titre, qui est le véritable protagoniste de la nouvelle, et celle-ci est entièrement construite pour mettre en avant, dans les ultimes pages, l'acte de cet homme qui fonde la problématique du récit. L'extermination de ses parents suscite chez le fils une haine inextinguible à l'égard des Allemands, de tout ce qui symbolise l'Allemagne. Dès lors, il n'aura de cesse qu'il n'ait accompli ce qu'il considère comme une vengeance personnelle à laquelle il ne peut se soustraire. En 1945, il est médecin à Paris. Appelé en urgence par le mari au 
chevet d'une jeune femme qui, à la suite d'une fausse couche, est en train de perdre tout son sang et risque de mourir, il entend la patiente murmurer quelques mots en allemand. Partagé entre son devoir de médecin et son désir de vengeance, il résoudra le dilemme en transfusant à la malade son propre sang, la sauvant ainsi d'une mort certaine. Au narrateur qui s'interroge, Lise précise la véritable signification de cet acte pour celui qui l'a commis : le médecin a certes sauvé sa patiente, mais le fils de déportés juifs a aussi " condamné » cette Allemande, cette "Aryenne ", à vivre, à vivre avec du sang juif dans les veines ! «Il a contaminé toute la race », conclut Lise lapidairement ( $K$, p. 163). Singulière réinterprétation, macabre retournement de l'une des composantes les plus délétères - la pseudo pureté du sang et donc de la race - de l'idéologie nationalsocialiste. Et si le narrateur - allemand - trouve l'idée "sublime", Lise, la juive, la qualifie de «folle ", l'idée d'un "malade », d'un «masochiste ", renvoyant ainsi dos à dos les assassins initiaux et le vengeur aveuglé par sa haine ${ }^{5}$. Certes, comme souvent chez Kleeberg, les dernières lignes de la nouvelle atténuent quelque peu la noirceur du sujet, mais le lecteur n'oubliera pas de si tôt cet acte de vengeance ni la manière dont il a été exécuté. Pouvoir et force de la littérature encore pour transcrire le réel. Art incomparable du nouvelliste dans la construction de son récit.

Ce même passé, mais selon des modalités différentes, resurgit brusquement à la mémoire de l'un des deux protagonistes de Liebes Brüderchen, Liebe Schwester, un autre médecin que l'une des expressions utilisées par l'étrange patiente venue lui demander de l'aide pour mettre fin à ses jours replonge brusquement près de cinquante ans en arrière. L'expression - une insulte des plus vulgaires proférée à son endroit par la vieille femme ( Sie ... Sie sind ja nichts weiter als ein ... ein liebes Brüderchen sind Sie!», K, p.39.) - est la même que celle qui lui avait été adressée jadis, en des circonstances bien différentes, par un officier nazi. Jeune étudiant en médecine à l'époque, il était sur le point d'être enrôlé dans la Wehrmacht. Mais accusé d'être homosexuel, il est arrêté et amené dans la cave d'un hôpital où il se retrouve face à un médecin militaire entouré de son équipe. Il s'agit pour cet officier médecin, appliquant à la lettre une théorie qu'il a élaborée et dont il n'est pas peu fier, de vérifier si l'homosexualité des personnes arrêtées est bien réelle, inscrite dans les gènes, auquel cas elles seront exterminées, ou si cette homosexualité n'est en quelque sorte qu'une erreur passagère, qu'un virus malencontreusement attrapé, au contact d'un juif par exemple, dès lors la 'maladie' serait curable, un séjour du 'malade' sur le front pourrait le 'guérir'! Pour opérer cette vérification, pratiquer ce test de conformité sexuelle en somme, l'officier médecin a engagé des prostituées par l'intermédiaire desquelles les personnes soupçonnées doivent prouver, devant l'officier et son équipe, qu'elles sont capables d'avoir des relations sexuelles avec des femmes. Au fur et à mesure que le médecin se remémore devant la vieille femme cet épisode de son passé, celle-ci, à sa grande surprise, complète et précise le récit qu'il lui fait en évoquant certains détails que le médecin avait oubliés : il se trouve qu'elle était à l'époque l'une des prostituées engagées par l'officier! « Les vieilles blessures ne se referment pas », dit le médecin, et la formulation allemande est plus suggestive encore : «Alte Messer schneiden tief » $(K$, p. 39). C'est en effet bien dans une blessure ancienne, profonde et encore ouverte que la mémoire sollicitée vient de porter le fer. Fouaillé de la sorte, le passé resurgit et vient interférer avec le présent. L'analepse narrative sert à la perfection la thématique ici traitée par le nouvelliste. Ce 11 février 1944 a été un moment décisif dans la vie du médecin, ce jour-là sa vie s'est jouée. Individu face à son destin donc. Expérience traumatisante d'un être revécue avec la même acuité quarante-cinq ans plus tard. Mais 
il s'agit aussi d'une histoire individuelle insérée dans l'histoire collective, dans l'Histoire allemande, dont la période la plus sombre, illustrée ici par l'un de ses aspects les plus sordides, une fois de plus est revisitée par un écrivain, né bien après la guerre, comme si, décidément, le passé n'en finissait pas de passer, passé obsessionnel, taraudant, térébrant dans l'univers mental des Allemands.

Le propre de la nouvelle est de mettre en œuvre une structure souvent antithétique, de jouer sur des oppositions fondamentales, de faire se frotter et se confronter les extrêmes. Une tension sous-tend le texte, déployée entre des pôles qui s'opposent. Dans la manière dont l'espace y est présenté - qui reflète la problématique de cette nouvelle - , Birth of the cool illustre semblable schéma. De réalité allemande il est de nouveau question, en 1988 puis en 1990 ; Kleeberg fournit donc, dans ce recueil publié pour la première fois en 1997, sa contribution - originale - à la "Wende-Literatur ». La nouvelle est organisée autour de trois rencontres entre le narrateur et l'un de ses parents éloignés, Rudolf. La première rencontre a lieu en 1988 à Paris où réside le narrateur. Rudolf, batteur dans un groupe de jazz et professeur de musique en Saxe, a obtenu l'autorisation d'aller à Stuttgart pour rendre visite à sa grand-mère malade, puis, grâce à un visa, il a poursuivi son voyage jusqu'à Paris. Emerveillé par la métropole occidentale, il va un soir en compagnie du narrateur au " New Morning ", la plus célèbre boîte de jazz de la capitale française. Jay Murray y joue, que Rudolf a rencontré au festival de jazz de Varsovie ; le musicien américain le reconnaît et l'invite à venir jouer sur scène avec lui, notamment un morceau extrait de l'album de Miles Davies, «Birth of the cool ». La deuxième rencontre se passe deux ans plus tard, après la chute du Mur. La situation financière de Rudolf est difficile, son groupe de jazz s'est dissous, les musiciens ne trouvaient plus d'engagement, il n'est plus professeur de musique, le père du narrateur lui propose de devenir courtier en assurances, ce qu'il se résout à faire. Le narrateur voit Rudolf une troisième fois l'année suivante, à l'occasion de l'anniversaire de son père; trois musiciens, "The Wizards ", ont été engagés pour faire danser les invités, Rudolf est convié à se joindre à eux, le groupe joue une valse. La symbolique des lieux joue à plein dans cette nouvelle, et l'évocation des deux Etats allemands, séparés par une frontière étanche, dit bien l'affrontement de deux espaces irréductibles et qui, en 1988, paraissaient l'être encore pour longtemps. Affrontement de deux systèmes politiques, sociaux, économiques, de deux idéologies, qui ont fait surgir des mentalités opposées dont la chute du Mur, en définitive, ne fera qu'aviver l'irréductibilité, comme le montre le père du narrateur, chantre arrogant du capitalisme triomphant de l'ex Allemagne de l'Ouest, par le jugement qu'il porte sur Rudolf et l'ex-RDA ${ }^{6}$. Mais si la nouvelle circonscrit de la sorte, en évoquant les deux Etats allemands, deux espaces clos, imperméables l'un à l'autre, Kleeberg montre aussi que cette frontière, en apparence parfaitement hermétique, est en réalité poreuse et que le bonheur n'est peut-être pas là où on attendait qu'il fût. Rudolf, par l'activité qu'il exerce jusqu'en 1990 et le lieu où il s'y adonne, d'une certaine manière en effet abolit cette frontière et réalise l'oxymore : jazzman en Saxe ; cette idée d'un joueur de jazz saxon fait plutôt rire dans la famille du narrateur, même si ce dernier est également fier de Rudolf. Le jazz n'est-il pas la musique venue de la puissance capitaliste abhorrée, tout en étant il est vrai aussi, à l'origine, le mode d'expression des esclaves noirs exploités dans les champs de coton, autres damnés de la terre? Le festival de jazz de Varsovie, auquel assiste Rudolf, n'est-ce pas un espace recroquevillé sur lui-même un instant - le temps que dure la manifestation - enfreint, pénétré par la musique venue d'un autre monde, du «monde libre »? La scène au « New Morning », elle aussi, réalise 
l'oxymore, abolit la frontière, fusionne symboliquement et le temps d'une soirée les deux espaces : le jazzman de Saxe qui retrouve dans ce temple parisien - occidental du jazz le jazzman américain rencontré à Varsovie ; deux lieux si différents, si éloignés, si séparés qui se fondent et se confondent en un lieu unique, celui de la musique, de cette musique-là. Du festival de Varsovie à la jam session de Paris. Le "New Morning " ou la jam de Jay et de Rudolf. Rudolf heureux, au comble du bonheur même.

La chute du Mur qui, non plus symboliquement mais dans la réalité des faits et non plus momentanément mais irréversiblement et pour toujours, semble-t-il, réunit en un seul les deux espaces allemands, crée dans l'existence de Rudolf, paradoxalement - mais l'est-ce vraiment pour Kleeberg? -, une autre frontière ou, plutôt, en inversant les valeurs, remet en cause ce qui fut jusqu'alors sa vie et détruit cette forme de bonheur qui était la sienne. L'ancien jazzman et professeur de musique est devenu un agent d'assurances acharné au travail, rentabilité capitaliste oblige. Dérisoire est la scène de l'anniversaire où, dans le trio engagé pour l'occasion, il prend la place du batteur pour jouer une valse. De la jam session au « New Morning» - lieu magique - où il jouait Miles Davies en compagnie de Jay Murray à l'air de valse joué par «The Wizards ", d'une musique l'autre, l'une, essentielle, historique, expression d'un art authentique, l'autre, jouée par un groupe ringard au nom ridiculement anglais qui, en dépit de ce nom, paraît condamné à ne se produire que sur des scènes de seconde zone : ce raccourci dit à lui seul l'itinéraire décevant de Rudolf. Kleeberg n'offre pas dans cette nouvelle une vision idéologiquement manichéenne, où la RDA serait présentée comme le contreexemple à bannir et la RFA comme le modèle idéal qui rendrait l'individu heureux. La question reste même posée de ce qui prévaut, de l'ex-RDA, son système et ses contraintes, mais où l'amateur de jazz Rudolf pouvait s'adonner à sa passion, ou de la nouvelle Allemagne (ré)unifiée dans laquelle l'ancienne partie occidentale impose avec arrogance à l'ex-partie orientale son mode de fonctionnement fondé sur la rentabilité et le profit, où désormais pour Rudolf la passion est rabaissée au rang de hobby bien éphémère.

Regard décapant que pose ici Kleeberg sur une autre réalité allemande.

\section{Instants décisifs}

Certains instants prélevés dans le flot d'une vie, certains instants seulement mais éclairés alors d'une lumière particulière, des moments intenses qui, s'ils ne font pas toujours basculer l'itinéraire des personnages qui les vivent, marquent toutefois leur existence, imprègnent parfois leur mémoire d'un signet ineffaçable, événements relatés ensuite, dans un récit rétrospectif, par le narrateur qui en a été l'acteur : telle est bien encore la configuration de nombre d'autres nouvelles composant le recueil. Ainsi de cette «brève amitié" (Eine kurze Freundschaft) liant les deux étudiants que sont le narrateur et Christian. Le campus universitaire, les salles de cours, les chambres d'étudiants, les parcs et la ville de Hambourg, une course effrénée à vélo par une nuit d'orage constituent les décors de cette complicité intense, quelques semaines durant, avant que Christian, qui a bénéficié d'une bourse d'études, ne se rende aux États-Unis et que les deux amis ne se perdent de vue. Six années plus tard, ayant écrit aux parents de Christian, le narrateur apprendra que son ancien camarade d'études était revenu des États-Unis au bout de deux ans et que, miné par l'alcool et la drogue, il s'était suicidé. Etudiant brillant, à l'existence brisée. 

échouent la nuit à l'accueil d'un hôpital, simulateur qui souhaite être hébergé, folle échappée de l'établissement où elle est soignée, vieil homme ne supportant plus la solitude chez lui depuis l'hospitalisation de son compagnon et venu le retrouver, accueilli pour la nuit par le médecin de garde qui lui taira encore l'espace de quelques heures la mort de son ami.

C'est un souvenir d'enfance, toujours ancré dans sa mémoire, que relate le narrateur de Der Herd : cette visite qu'il a faite avec ses parents à huit ans, en Suisse, à une tante qu'il ne connaissait pas et qui, atteinte d'un cancer, était sur le point de mourir. S'il n'a pas oublié l'odeur écoeurante d'urine et d'eau de Cologne dans la chambre de la malade, le visage émacié où il recherchait la présence de la mort, il se souvient tout autant des paroles attentionnées qu'elle avait eues à son égard, du livre donné par l'oncle, comme de la fillette furtivement aperçue dans le jardin d'à côté. Mais il n'a pas oublié non plus «la cuisinière » électrique neuve, offerte par la tante et rapportée dans le coffre de la voiture, volontairement salie par son père avant le voyage retour afin de la faire passer pour usagée à la douane et payer ainsi moins de taxes! Premier contact de l'enfant avec la mort, découverte de l'hypocrisie et de la rapacité des adultes aussi; autant d'expériences déterminantes dans une vie.

Le ton est semble-t-il plus léger dans Die zwei Leben des Dominik D, puisqu'il s'agit d'une variation sur le thème de l'astrologie. Est conté le destin de deux personnes que tout sépare, mais qui sont nées le même jour, au même endroit, à quelques minutes près au même moment. Mais la tonalité se fait plus sombre dans les ultimes lignes de la nouvelle qui évoquent la mort de Dominik $D$ après que son alter ego zodiacal eut subi le même sort. Prédestination, mystérieuses connexions entre les vies des êtres, hasard ?

C'est sa rencontre, fortuite, avec une jeune fille, bien des années après qu'elle s'est produite, que relate le narrateur de Kebab ist überall. Brève rencontre qui ne dura que trois jours et resta sans suite. Mais relation intense, de plénitude amoureuse entre les deux personnages, dont la complicité intellectuelle et physique est parfaite durant ce bref moment. Le titre - très énigmatique - de la nouvelle reproduit une formule qu'un troisième protagoniste utilise pour un oui ou pour un non, mi-juron, mi-expression désabusée ou fataliste, ce chauffeur de taxi turc, réfugié politique en Suisse, persuadé d'être épié et suivi en permanence par les services secrets de son pays d'origine, qui convoie les amoureux dans la ville de Lugano, complice discret de leur passion, bon génie oriental veillant sur eux. Il meurt un jour dans un accident de voiture alors qu'il se rend à Milan, pensant échapper ainsi à un tueur chargé de l'abattre qu'il prétend avoir aperçu. Sa mort se produit peu de temps après la séparation définitive des deux amants, comme s'il y avait une mystérieuse relation entre les deux événements, comme si le bon génie qui avait veillé sur leur amour ne devait pas survivre à celui-ci. Dans cette nouvelle, où il sacrifie aussi - non sans humour décalé ou distance légèrement ironique - au topos du " voyage en Italie », du voyage vers le sud et ses charmes en tout cas - le narrateur est originaire de Hambourg, se rend en Italie, et s'arrête cette fois en Suisse après avoir croisé dans le train le chemin de la jeune fille -, Kleeberg propose une réflexion douce-amère sur la destinée humaine, faite de rencontres qui peuvent être fortes mais demeurent aussi comme inéluctablement éphémères, sur le hasard, la fatalité, sinon le fatalisme.

31 Deux vies qui, irréversiblement, bifurquent, et cette fois pour le meilleur des deux protagonistes semble-t-il : telle est, schématiquement présentée, la configuration de 
Der Kommunist vom Montmartre, nouvelle entièrement construite sur un effet de contrastes extrême. On est en 1935 quand s'ouvre le récit. Gaspard Morand, membre du comité central du Parti Communiste Français, est chargé de constituer une délégation qui doit se rendre à Moscou. La délégation doit comporter un représentant de tous les peuples opprimés à l'époque par « l'impérialisme français ». Si Morand n'a aucun mal à trouver un Algérien, un Vietnamien, un Polynésien ou un «mulâtre des Caraibes », ses recherches restent infructueuses dès lors qu'il lui faut proposer le nom d'un représentant de «l'Afrique Noire exploitée " qui soit aussi membre du parti. En définitive, il n'a plus d'autre solution que de passer une petite annonce - dans le Figaro! -, au contenu nécessairement déguisé, ne révélant rien du véritable objectif du voyage. Une seule personne se présente: un certain Di Lammermoor, un noir en effet, mais artiste de variétés, homosexuel, se livrant d'ordinaire à un numéro de travesti dans un cabaret de Montmartre, et aux antipodes bien entendu des préoccupations habituelles des militants du parti. Au plus grand étonnement de Morand, Di Lammermoor conçoit ce qu'on lui propose comme un nouveau numéro pour lequel il se fait fort de déployer toute la palette de ses talents. En quelques semaines, l'artiste de variétés est initié par un jeune ouvrier travaillant sur les chaînes de montage de l'usine Citroën, François, avec lequel il ne tarde pas à se lier d'amitié, aux rudiments de l'idéologie communiste. En dépit des craintes de Morand, les cérémonies moscovites, au cours desquelles l'artiste joue son rôle à la perfection, se déroulent au mieux. Une fête rassemblant toutes les délégations est organisée le dernier soir de la visite, un orchestre fatigué joue. Soudain la musique se tait, apparaissent alors sur la scène Mistinguett, alias Di Lammermoor, avec le costume approprié, puis François en un Maurice Chevalier plus vrai que nature, chacun entonnant l'une des chansons du répertoire des deux artistes. Ce n'est pas l'épisode final de la nouvelle. Une dernière scène montre le jeune François renonçant à son métier et au parti pour tenter de faire carrière au cabaret, et Di Lammermoor entrant au parti, en payant de son cachet sa cotisation, pour, comme il le dit, apporter son soutien à «la lutte» et s'efforcer de colorer un peu la grisaille du monde.

Le burlesque qui surgit d'abord de la personnalité et du comportement de Di Lammermoor puis du choc saisissant entre ces deux univers diamétralement opposés est à l'évidence à l'œuvre dans la nouvelle. Mais outre cette dimension burlesque, ou peut-être grâce à elle, naît aussi une réflexion, amère ou désabusée, de l'auteur sur le monde et l'existence humaine. Significative à cet égard est une remarque de l'artiste, alors que François s'efforce de le convaincre que ce voyage n'est nullement une tournée de music-hall : « Mon p'tit, le monde entier est un théâtre, et chacun joue son rôle. Peu m'importe le titre de la pièce à partir du moment où je suis sous les projecteurs. » $(K$, p. 18)

On reconnaît ici, même s'il est revu et corrigé non sans un certain cynisme par l'artiste, l'un des plus anciens lieux communs littéraires, celui du monde comme théâtre sur la scène duquel chacun joue un rôle. L'existence de Di Lammermoor n'est qu'un jeu de rôles permanent, sur la scène comme dans la vie. Mesurée à l'aune de cette réflexion générale de l'artiste - de Kleeberg ? - sur l'existence humaine, que représente dès lors la vie d'un Morand ou d'un François? Quel sens donner au combat que mène avec conviction, persuadé d'être un acteur de l'Histoire, un ouvrier, militant communiste affiché ? Tout n'est-il en définitive qu'apparence et faux-semblant? 

la suite des décisions que prennent Di Lammermoor et François, ce sont deux existences qui changent du tout au tout. Et ces décisions, lorsqu'on lit comment les deux hommes les justifient, paraissent avoir été prises avec toute la gravité requise. François, l'ouvrier de Citroën, le membre du parti, le militant convaincu et actif, qui renonce à son métier, quitte le parti pour se consacrer au théâtre, comme s'il avait découvert au contact de l'artiste et au cours du voyage le véritable sens de sa vie. Di Lammermoor, l'artiste de music-hall à la célébrité toute relative, qui lors de ce même voyage a pris conscience de la justesse du combat que mènent Morand et ses camarades, a observé la grisaille, la misère et la tristesse de la vie chez le "grand frère " soviétique et qui, dès lors, par son adhésion au parti et par le rôle qu'il entend désormais y jouer, souhaite insuffler à cette vie un peu plus de couleur et de gaieté. Aurait-il lui aussi, de la sorte, trouvé un sens à sa vie?

Inversion de rôles donc: l'ouvrier qui devient artiste, l'artiste qui entre au parti des ouvriers. Inversion de références : François s'extirpant des codes qui étaient jusqu'alors les siens et encadraient sa vie pour un univers qui refuse les normes communément admises. Di Lammermoor, celui qui, parce qu'il est un artiste, est par définition hors normes, se glissant dans un moule où, par définition, règne la norme. Inversion d'espaces : de la chaîne à la scène, du cabaret au parti.

\section{Art de la nouvelle}

Un passé allemand qui n'en finit pas de passer, des destins individuels qui, un jour, par le fait d'un événement ou d'une rencontre, basculent, expériences radicales, hapax existentiels à l'issue desquels rien ne sera plus comme avant, souvenirs qui sont autant d'empreintes indélébiles, vies à la dérive : les nouvelles de Kleeberg ${ }^{7}$ proposent des variations sur ces thèmes essentiels qui, réunies, élaborent une réflexion douce-amère, ironique ou désabusée, non dénuée d'humour aussi parfois, sur l'existence humaine, sur la quête identitaire de chacun, sur le sens que l'on peut donner à sa vie. Littérature et réalité donc.

$\mathrm{Au}$ concours de la petite ville autrichienne, ces nouvelles n'entreraient assurément pas dans la catégorie des textes expérimentaux. On peut dire leur facture "classique », elles s'inscrivent dans une longue tradition, on y repère aussi nombre de traits caractéristiques, de lois organisatrices qui fondent et fixent le genre. La brièveté relative - de ces textes détermine des spécificités, mais à l'intérieur des limites imposées se lisent également d'infinies potentialités. Les récits, construits sur un canevas précis dont toutes les composantes s'articulent fortement les unes par rapport aux autres, se concentrant sur des épisodes essentiels, structurés en une progression rigoureuse, se déroulent de façon linéaire, d'une situation initiale à une situation finale qui modifie radicalement la première, acmé et chute qui sont lieu et moment stratégiques où l'intrigue s'achève et se parachève. Les protagonistes, par définition condamnés à un temps fragmenté, discontinu, existent le plus souvent dans la rupture, paraissent n'avoir d'existence que par rapport à la situation dans laquelle ils sont plongés, qu'en relation avec la trame des événements qui ponctuent leur itinéraire. Le segment temporel qui les montre agir correspond au temps de l'aventure qu'ils vivent, parenthèse inscrite dans des vies, moments spécifiques, pour le meilleur ou pour le pire, un temps hors du temps habituel, comme une uchronie significative. On ne peut 
qu'être attentif, enfin, à la manière dont Kleeberg traite l'espace dans ses nouvelles. Si la fonction référentielle, traditionnellement soulignée à propos des décors, qui consiste à situer le cadre, à faire voir et à faire vrai, à donner au récit l'épaisseur du réel, est bien là, on est surtout sensible - et dans ce domaine aussi se déploie tout l'art du nouvelliste - au lien étroit entre espace et signification; l'on perçoit comment les éléments constituant l'espace, leurs résonances disséminées dans l'œuvre, s'insèrent dans le flux narratif comme véritables articulations et non comme simples éléments ornementaux, l'on apprécie comment signe et sens fusionnent.

« Literatur » donc, au sens fort du terme, que ces nouvelles de Michael Kleeberg.

\section{NOTES}

1. Principales traductions :Yves Simon, Die Drift der Gefühle. Halle, Mitteldeutscher Verlag, 1994.Xavier Hanotte, Das Bildnis der Dame in Schwarz. München, Deutscher Taschenbuch Verlag, 1996. Von verschwiegenem Unrecht. München, Deutscher Taschenbuch Verlag, 1999.Pascal Bruckner, Diebe der Schönheit. Berlin, Aufbau, 1998.Paule Constant, Vertrauen gegen Vertrauen. Frankfurt/Main, Frankfurter Verlagsanstalt, 1999. Sex und Geheimnis. Frankfurt/Main, Frankfurter Verlagsanstalt, 2003.Virginie Despentes, Pauline und Claudine. Reinbeck, Rowohlt, 2001.Jules Barbey d'Aurevilly, Finsternis. Bremen, Manholt, 1999.Marcel Proust, Combray. München, Liebeskind, 2002. Eine Liebe Swanns. München, Liebeskind. 2004.

2. Der saubere Tod. Roman. München, Schneekluth. 1987.Proteus der Pilger. Roman. Halle, Mitteldeutscher Verlag, 1993.Ein Garten im Norden. Roman. Berlin, Ullstein, 1998.Der König von Korsika. Roman. Stuttgart, Deutsche Verlags-Anstalt, 2001. Traduction française: Le Roi de Corse. Paris, Flammarion, 2004.Das Tier, das weint. Libanesisches Reisetagebuch. Stuttgart, Deutsche Verlags-Anstalt, 2004.

3. Böblinger Brezeln. Stories. München, Schneekluth, 1984.Barfuß. Novelle. Köln, Kiepenheuer \& Witsch, 1995. Traduction française: Pieds nus. Paris, Denoël, 1996.Der Kommunist vom Montmartre und andere Geschichten. Köln, Kiepenheuer \& Witsch, 1997. L'édition de référence dans cette contribution est: München, Deutscher Taschenbuch Verlag, 2002. Edition identifiée désormais par $K$, suivi du numéro de la page.Au moment où ces lignes sont écrites, il n'existe, à ma connaissance, sur les oeuvres de Michael Kleeberg, et notamment sur les nouvelles, que des recensions dans des journaux ou revues; il n'y a pas d'étude d'ensemble.

4. On ne peut s'empêcher ici de songer aux poèmes écrits par Rose Ausländer, dans d'autres « caves », celles de Czernowitz, poèmes qui disent, plus de cinquante ans auparavant, une autre réalité d'une autre guerre. Pouvoir du langage poétique là aussi.

5. «Das ist, das ist wirklich sublim als Idee...Verrückt ist es. Krank. Masochistisch, sagte Lise abfällig. » $K$, p. 163.

6. «Aber daß die Zeiten vorübergehend härter werden und die Leute jetzt mal nach vierzig Jahren den Ernst des Lebens sehen und lernen müssen, was arbeiten heißt, das ist doch logisch. Wir tun unser Teil, aber die müssen eben auch das ihre tun und anfangen, ein bißchen Initiative zu entwickeln. Wir stecken da 100 Milliarden rein, und wir können das ja zum Glück auch... » $K$, p. 83. 
7. Voir, dans la même perspective, les 5 autres nouvelles constituant ce recueil et qui n'ont pu être analysées ici.

\section{RÉSUMÉS}

Le présent travail se propose d'analyser le second volume de nouvelles (Der Kommunist vom Montmartre und andere Geschichten, 1997), publié par Michael Kleeberg. Tant sur le plan des thèmes abordés que sur celui des techniques d'écriture mises en œuvre, ces «histoires » montrent un auteur, en l'occurrence ici un nouvelliste, au sommet de son art. Un passé allemand qui n'en finit pas de passer, des destins individuels qui, un jour, par le fait d'un événement ou d'une rencontre, basculent, des souvenirs qui sont autant d'empreintes indélébiles, des vies à la dérive : les nouvelles de Kleeberg proposent des variations sur ces thèmes essentiels qui, réunies, élaborent une réflexion douce-amère, ironique ou désabusée, non dénuée d'humour aussi parfois, sur l'existence humaine, sur la quête identitaire de chacun, sur le sens que l'on peut donner à sa vie. On repère par ailleurs dans ces textes nombre de traits caractéristiques, de lois organisatrices qui, dans la configuration de l'action, le traitement de l'espace et du temps, la caractérisation des personnages notamment, fondent le genre de la nouvelle.

Ziel der vorliegenden Arbeit ist die Sammlung Der Kommunist vom Montmartre und andere Geschichten (1997) von Michael Kleeberg zu untersuchen. Die in diesen «Geschichten» behandelten Themen sowie Kleebergs Erzähltechnik zeigen, daß wir es hier schon mit einem hervorragenden Novellisten zu tun haben.

Eine deutsche Vergangenheit, die eben nicht vergeht, Menschen, die eines Tages wegen eines bestimmten Ereignisses oder einer Begegnung aus ihrem bisherigen Dasein hinausgestoßen werden und in eine für sie völlig neue Situation geraten, unauslöschliche Erinnerungen, ziellos gewordene Existenzen: Aus all diesen Themen ergibt sich eine bittersüße, ironische, desillusionierte, manchmal auch humorvolle Reflexion über die menschliche Existenz, die Identitätssuche des Individuums, den Sinn, den jeder seinem Leben zu geben vermag. Darüberhinaus lassen sich in diesen « Geschichten » relevante formale Merkmale herausarbeiten, welche die Novelle als Gattung konstituieren, u.a. im allgemeinen Aufbau der Handlung, in der Darstellung des Raums und der Zeit und in der Charakterisierung der Figuren.

\section{INDEX}

Mots-clés : passé allemand, quête identitaire

oeuvrecitee Böblinger Bretzel, Barfuß, Der Kommunist von Montmartre und andere Geschichten

\section{AUTEURS}

\section{ALAIN COZIC}

Université de Toulouse 2 